\title{
Detecting Current Noise with a Josephson Junction in the Macroscopic Quantum Tunneling Regime
}

\author{
J.T. Peltonen ${ }^{1}$, A.V. Timofeev ${ }^{1,2}$, M. Meschke ${ }^{1}$, and J.P. Pekola ${ }^{1}$ \\ ${ }^{1}$ Low Temperature Laboratory, Helsinki University of Technology, \\ P.O. Box 3500, 02015 TKK, Finland \\ ${ }^{2}$ Institute of Solid State Physics, Russian Academy of Sciences, \\ Chernogolovka, 142432, Russia
}

\begin{abstract}
We discuss the use of a hysteretic Josephson junction to detect current fluctuations with frequencies below the plasma frequency of the junction. These adiabatic fluctuations are probed by switching measurements observing the noise-affected average rate of macroscopic quantum tunneling of the detector junction out of its zero-voltage state. In a proposed experimental scheme, frequencies of the noise are limited by an on-chip filtering circuit. The third cumulant of current fluctuations at the detector is related to an asymmetry of the switching rates.
\end{abstract}

PACS numbers: 82.25.Cp, 05.40.-a, 72.70. $+m$

\section{INTRODUCTION}

Complete description of charge transport through a mesoscopic conductor requires knowledge of the probability distribution of current through the conductor. In general, such distributions are characterized not only by the average current and variance, but also by higher order moments. This study of fluctuations has attracted intense theoretical activity in the recent years, and powerful frameworks such as full counting statistics (FCS) have been formulated.12 Starting from fundamental microscopic theories, moment-generating functions are known for several systems such as tunnel junctions and quantum dots. Experimental investigation of the fluctuations beyond the variance is, however, not so well-established. Experiments observing higher moments of current or voltage have remained difficult and time consuming as filtering and bandwidth requirements are hard to ful- 


\section{J.T. Peltonen et al.}

fill - detection of higher moments of fluctuations is typically characterized by very weak signals and wide bandwidth measurements performed at low temperatures where thermal effects are suppressed.

The first measurements 3 of the third moment of current fluctuations across a voltage-biased tunnel junction support the theoretical concepts, although the use of conventional amplifiers and mixers requires long averaging times. Furthermore, the electrical environment of the conductor can significantly affect the measured statistics ${ }^{4}$ In a more recent measurement, $\frac{5}{5}$ the use of a slightly different detection scheme provides a more direct access to fluctuations of voltage up to the third moment.

Among the growing number of experimental findings, remarkable results have been obtained using real-time detection of single electrons, ${ }^{6 / 7}$ which can be utilized in the measurement of full counting statistics of electron transport. Recently, even further progress was accomplished in a measurement of the distribution of tunneling current through a quantum dot ${ }^{8}$ These innovative approaches are examples of on-chip detection of fluctuations, which is the design goal of future experimental schemes as well. In this way, one can perform faster measurements on a wider bandwidth and avoid the problems with remotely connected amplifiers.

On the other hand, the above techniques based on direct counting of electrons are best suited up to moderate frequencies and extremely low current levels in the pA range and below. Other experimental approaches are needed at considerably higher currents and for frequencies in the range of several $\mathrm{GHz}$, indispensable for the characterization of many involved processes. One solution is the use of Josephson junctions (JJs) as noise probes. With small low-capacitance junctions, the sensitivity of the Coulomb blockade can be used to characterize voltage fluctuations generated by a mesoscopic conductor ${ }^{9 / 10}$ Another possibility is the use of larger current-biased junctions as threshold detectors $\frac{11 \mid 12}{}$ to probe the higher moments of current noise. In this case, switching rate of the junction from the supercurrent to the normal state depends strongly on the fluctuations in the bias current. The applicability of such a JJ detector to measure shot noise has been demonstrated, $\frac{13}{b}$ but convincing experimental results concerning the higher moments of fluctuations have not yet been reported.

A JJ appears to be quite an attractive threshold detector at the first view: it detects with a certain probability a current exceeding a given threshold, and switches consequently from the superconducting state to a well

detectable normal state. A more detailed description of the dynamics of the junction acting as a noise detector reveals several mechanisms though: apart from the usual crossover from thermal activation (TA) to macroscopic quantum tunneling (MQT) as a function of temperature, the response of a 


\section{Detecting Current Noise with a Josephson Junction}

hysteretic Josephson junction to current noise depends inherently on both the spectrum and distribution of the fluctuations. In the TA regime, the switching of the junction occurs by thermally activated escape over a high potential barrier. On the contrary, at lower temperatures the escape results as the phase of the superconducting order parameter over the junction tunnels quantum mechanically through the barrier. These processes are affected by current fluctuations, and the influence of noise on the escape dynamics has been correspondingly analyzed for junctions in different parameter and temperature regimes. $\frac{13|14| 15 \mid 16}{}$ For example, high-frequency noise leads to the notion of an effective temperature $T^{*}$ of the detecting junction, which often exceeds the superconducting transition temperature $T_{\mathrm{c}} \underline{13}$

In this work, we first characterize in general the influence of current fluctuations on a Josephson junction initially in the regime of macroscopic quantum tunneling. Consequently, we discuss the sensitivity of a JJ when detecting the higher moments of high-frequency noise using switching measurements. This leads us to investigate the influence of weaker low-frequency fluctuations that can be considered adiabatic in view of the quantum dynamics of the detector. Since achieving suitable sensitivity to fluctuations in this certain frequency range depends strongly on the electrical environment of the detector, we discuss the requirements for a feasible experimental detection scheme such as increasing the plasma frequency $\omega_{\mathrm{p}}$ of the detector junction or including filtering to limit the accessible noise bandwidth to the desired range.

\section{INFLUENCE OF CURRENT FLUCTUATIONS ON A JOSEPHSON JUNCTION DETECTOR IN DIFFERENT FREQUENCY REGIMES}

In this work we concentrate on discussing how a current-biased hysteretic Josephson junction in the regime of macroscopic quantum tunneling can be used to characterize weak fluctuations in its bias current. Such a junction can act as a sensitive on-chip detector of current noise since the tunneling rate of the phase depends exponentially on current fluctuations. Under certain conditions this sensitivity may allow to distinguish non-Gaussian features of the fluctuations, which forms a central part of the discussion to follow.

In a general situation when the detector junction is part of an electrical circuit, these fluctuations of bias current may be of several origins. First, equilibrium noise is always present in the circuit, even when external current and voltage sources are turned off. For a general linear electrical 


\section{J.T. Peltonen et al.}

circuit, the spectral density $S_{I}(\omega) \equiv \int_{-\infty}^{\infty} \mathrm{d} t\langle I(t) I(0)\rangle \exp (i \omega t)$ of these normally distributed equilibrium fluctuations is determined by the fluctuationdissipation theorem. As in the previous formula, assuming the distribution of the fluctuations to be stationary, the noise power at the detector junction at frequency $\omega$ is given by ${ }^{2}$

$$
S_{I}^{\mathrm{env}}(\omega)=2 \hbar \omega \operatorname{Re}[Y(\omega)]\left(\operatorname{coth} \frac{\hbar \omega}{2 k_{\mathrm{B}} T}+1\right),
$$

where $T$ is the temperature and $Y(\omega)$ is the frequency-dependent admittance of the electrical circuit seen from the detector. At low frequencies or high temperatures with $\hbar \omega \ll k_{\mathrm{B}} T$, this reduces to the familiar expression for white thermal noise, $S_{I}^{\text {env }}(\omega)=4 G k_{\mathrm{B}} T$ with $G \equiv \operatorname{Re}[Y(\omega \approx 0)]$ denoting an effective conductance. Likewise, at the opposite limit we recover the expression for high-frequency quantum noise.

Besides these equilibrium fluctuations, generally non-Gaussian nonequilibrium noise may be present in the circuit as well. For example, in a circuit containing a voltage-biased tunnel junction in the normal state, nonequilibrium shot noise arises from the stochastic tunneling of discrete charges through the junction.

When both equilibrium and nonequilibrium fluctuations affect the detector, the total noise power at the detector junction is obtained as the incoherent sum of the different contributions: $S_{I}(\omega)=S_{I}^{\mathrm{env}}(\omega)+S_{I}^{\mathrm{shot}}(\omega)$. The noise power related to the second cumulant of the shot noise at a finite temperature in the low-frequency limit is given by ${ }^{17} S_{I}^{\text {shot }}=2 e F_{2} \bar{I}_{\mathrm{N}} \operatorname{coth}\left(e V / 2 k_{\mathrm{B}} T\right)$, where $\bar{I}_{\mathrm{N}}=V / R_{\mathrm{N}}$ is the average current through the junction with resistance $R_{\mathrm{N}}$ at the bias voltage $V$. Furthermore, $F_{2}$ is the Fano factor of the second moment, relating the microscopic transport properties of the conductor to the measurable noise. For a normal tunnel junction with low barrier transparency, $F_{2}=1$. Similarly, the third cumulant of these nonequilibrium fluctuations has in the same limit the non-zero theoretical value $C_{3}=F_{3} e^{2} \bar{I}_{\mathrm{N}}$, where $F_{3}$ is the Fano-factor of the third moment!18|19 The third cumulant describes the first-order deviations or asymmetry as compared to a Gaussian distribution, being therefore one of the first quantities to measure when investigating the noise properties of any mesoscopic conductor beyond the variance of current.

\subsection{Dynamics of a Josephson junction}

The above expressions or their extensions give the spectral densities of fluctuations at an arbitrary frequency and temperature. The response of the 


\section{Detecting Current Noise with a Josephson Junction}

detecting junction to current noise at different frequencies can be explained by describing its dynamics using the common resistively and capacitively shunted junction (RCSJ) - model. ${ }^{20|21| 22}$ To be more specific, let us consider a JJ with critical current $I_{\mathrm{c}}$ and capacitance $C$ under the influence of an external bias current $I(t)=I_{0}+\delta I(t)$. Here $I_{0}$ is the time-independent average bias current and $\delta I(t)$ describes the fluctuations. Classically, for $I \leq I_{\mathrm{c}}$ the JJ stays in the supercurrent state and the voltage over the junction is zero. On the other hand, a bias current $I>I_{\text {c }}$ causes it to switch to the resistive state, leading to a finite voltage of at least twice the superconducting energy gap $\Delta$ to develop over the junction.

In the RCSJ-model, such a JJ is described in terms of a parallel combination of a capacitor with capacitance $C$, an ideal tunnel junction with critical current $I_{\mathrm{c}}$ and by a shunt resistance $R$. The current-phase relation of the tunnel junction follows the Josephson relation $I=I_{\mathrm{c}} \sin \varphi$, where $\varphi$ is the phase difference of the superconducting order parameter over the junction, related to the voltage by $V=\hbar \dot{\varphi} / 2 e$.

For most of the discussion to follow, the electrical environment of the junction can be described by the effective circuit illustrated in Fig. 1. Here the current source $I_{\text {eff }}$ contains both a constant bias component $I_{0}$ and a time-dependent part related to $\delta I(t)$, whose statistical properties are determined by the shot noise source.

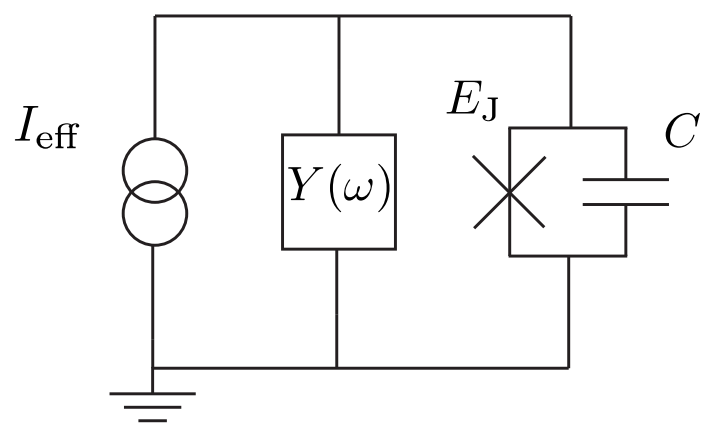

Figure 1. An effective circuit model for a JJ used as a noise detector. Nonequilibrium fluctuations are included in the properties of the effective current source producing a current $I_{\text {eff }}$. Electrical environment of the junction is described by the admittance $Y(\omega)$ responsible for equilibrium noise.

For small low-capacitance junctions the charging energy $E_{\mathrm{C}} \equiv e^{2} / 2 C$ is

not negligible, leading to a quantum mechanical description of the junction ${ }^{23}$ as the charge $q$ and phase $\varphi$ over the junction are conjugate variables satisfying $[\varphi, q]=2 i e$. In the limit of low dissipation, the junction is described 


\section{J.T. Peltonen et al.}

by the RCSJ-Hamiltonian

$$
\mathcal{H}=\frac{q^{2}}{2 C}+U(\varphi)=\frac{q^{2}}{2 C}-E_{\mathrm{J}}\left[\cos \varphi+\frac{I(t)}{I_{\mathrm{c}}} \varphi\right],
$$

where $E_{\mathrm{J}} \equiv \hbar I_{\mathrm{c}} / 2 e$ is the Josephson coupling energy. With the above commutation relation in mind, the behavior of a junction with $E_{\mathrm{C}} \ll E_{\mathrm{J}}$ can therefore be visualized as that of an imaginary quantum particle with mass $m=(\hbar / 2 e)^{2} C$ moving in a tilted cosine potential $U(\varphi)$. At low temperatures, the $\varphi$-particle is localized in one of the wells of this 'washboard' potential, performing oscillations at a local minimum at the plasma frequency $\omega_{\mathrm{p}}=\sqrt{8 E_{\mathrm{J}} E_{\mathrm{C}} \varphi_{0}} / \hbar$. Here the parameter $\varphi_{0}$ is related to the bias current $I$ changing the tilt of the potential by $\varphi_{0} \equiv \sqrt{1-\left(I / I_{\mathrm{c}}\right)^{2}}$.

Localization of the phase particle corresponds to the junction being in the supercurrent state. The state is, however, metastable, and the phase can escape from the potential well by macroscopic quantum tunneling through the barrier. To estimate the rate of this tunneling process, for currents $I \lesssim I_{\mathrm{c}}$ one well-barrier section of the potential $U(\varphi)$ can be approximated by a cubic parabola ${ }^{24}$

$$
U(\varphi) \simeq \frac{3}{4} \Delta U\left(\frac{\varphi}{\varphi_{0}}\right)^{2}\left(1-\frac{1}{3} \frac{\varphi}{\varphi_{0}}\right)
$$

where $\varphi$ is now measured from a minimum of the potential and $\Delta U$ is the height of the barrier given by

$$
\Delta U=\frac{2}{3} E_{\mathrm{J}} \varphi_{0}^{3} \simeq \frac{4 \sqrt{2}}{3} E_{\mathrm{J}}\left(1-\frac{I}{I_{\mathrm{c}}}\right)^{\frac{3}{2}} .
$$

Likewise, the tunneling rate $\Gamma(I)$ is obtained as

$$
\Gamma(I) \equiv A(I) e^{-B(I)}=12 \sqrt{6 \pi} \frac{\omega_{\mathrm{p}}}{2 \pi} \sqrt{\frac{\Delta U}{\hbar \omega_{\mathrm{p}}}} \exp \left(-\frac{36}{5} \frac{\Delta U}{\hbar \omega_{\mathrm{p}}}\right) .
$$

This follows from the treatment of tunneling out of a metastable cubic potential well in the semiclassical limit together with negligibly low dissipation. 24 Such a situation arises for small values of the real part of the effective shunting admittance $Y(\omega)$, corresponding to a high quality factor $Q \equiv$ $\omega_{\mathrm{p}} C / \operatorname{Re}[Y(\omega)]$.

Assuming that a constant current $I$ is applied for a time $\Delta t$, the finite decay rate $\Gamma(I)$ leads to the probability

$$
P(I)=1-\exp (-\Gamma(I) \Delta t)
$$




\section{Detecting Current Noise with a Josephson Junction}

for the phase to escape from the potential well. After tunneling, the particle starts running down the potential hill. For a hysteretic junction with $Q \gg 1$, this corresponds to the junction actually switching to the finite voltage state since the particle will not be trapped again into a local minimum until the bias current is lowered below the retrapping current close to zero. Hence, the quantum tunneling can be experimentally observed by applying repeated current pulses of constant height $I_{0}$ and length $\Delta t$ and recording the number of times the junction has switched to the normal state. Different values of $I_{0}$ then yield an escape probability histogram $P\left(I_{0}\right)$ to be compared with Eq. (6).

At higher temperatures, the MQT process is no longer the dominant way of escaping from the metastable well. Instead, the energy levels of the well have approximately thermal populations and the particle can escape over the potential barrier by thermal activation (TA) characterized by the $\operatorname{rate}^{24}$

$$
\Gamma_{\mathrm{T}} \simeq \frac{\omega_{\mathrm{p}}}{2 \pi} \exp \left(-\frac{\Delta U}{k_{\mathrm{B}} T}\right)
$$

Here $\Delta U$ is again the height of the barrier introduced in Eq. (4).

\subsection{Effect of noise on escape characteristics}

The above view of the dynamics of a JJ allows us to immediately distinguish a few different frequency regimes in terms of the response of the detecting junction to bias current fluctuations. To see this, let us suppose that the switching rate of the detector junction in the presence of noise is experimentally determined using the principle presented in Fig. 2, In more detail, we assume that $N$ constant bias current pulses of height $I_{0}$ and length $\Delta t$ injected in a time $\Delta t_{I_{0}}$ are used to obtain each single point on an escape probability histogram $P\left(I_{0}\right)$. The number $N$ is determined by the desired limit on the statistical error in the measurement. Since the total bias current $I(t)=I_{0}+\delta I(t)$ now contains a fluctuating part, the result is an average escape histogram differing from the ideal curve measured without the fluctuations $\delta I(t)$. Then, depending on the frequency content of the fluctuating current $\delta I(t)$, the pure MQT or TA rate [cf. Eqs. (5) and (17)] is modified as illustrated by the guidelines of Fig. 3, showing the typical behavior of the junction and the relevant characteristic frequencies.

For noise with frequencies well between $\Delta t_{I_{0}}^{-1}$ and $\Delta t^{-1}$, the detector junction is effectively biased by a constant current $I_{0, j}$ during the $j$ :th pulse. Consequently, the escape probability is approximately determined as an average over the distribution of $I_{0, j}$ probed by the detector when injecting 


\section{J.T. Peltonen et al.}

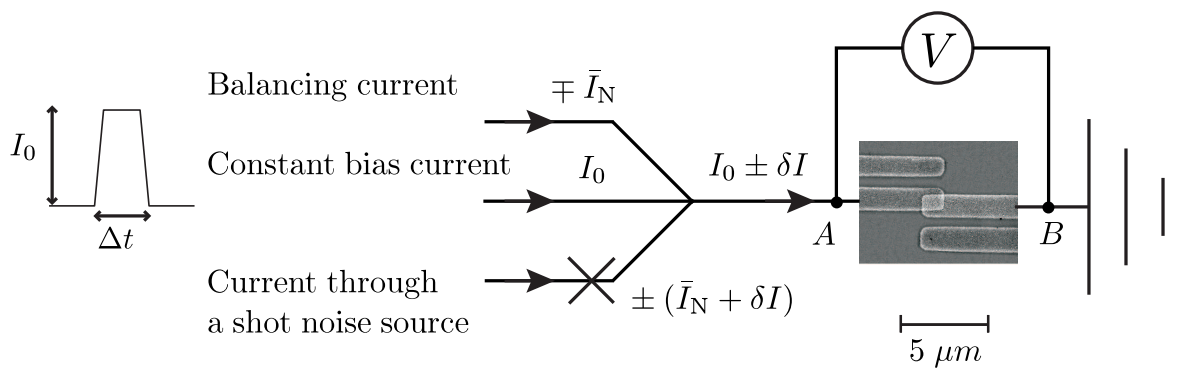

Josephson junction detector

Figure 2. A general measurement scheme to detect current noise with a JJ, showing the ideal flow of currents and a micrograph of a typical $\mathrm{Al}-\mathrm{AlO}_{\mathrm{x}}-$ Al JJ manufactured using electron beam lithography and 2-angle shadow evaporation. In a typical measurement, an average current $\bar{I}_{\mathrm{N}}$ is first injected through the noise source (a mesoscopic scatterer), whose temporal current can then be written as $\bar{I}_{\mathrm{N}}+\delta I(t)$. The DC component of this current is returned through the upper arm in the figure. In practise, this is achieved by injecting a balancing current $-\bar{I}_{\mathrm{N}}$ through the upper line. As a result, ideally only the fluctuating current $\delta I(t)$ flows through the detector junction located between points $A$ and $B$ in the figure. This balance of currents is verified by monitoring the average current through the detector. For a suitably designed circuit, the fluctuations $\delta I(t)$ pass mainly through the detector and do not leak back through the biasing lines. Finally, the escape rate of the detector $\mathrm{JJ}$ in the presence of the fluctuating current $\delta I(t)$ is measured by injecting $N$ pulses of constant height $I_{0}$ and length $\Delta t$ through a third line (middle arm in the figure). The $I_{0}$-pulses have long leading and trailing edges to ensure that the detector responds adiabatically to them. As a result of this three-fold current injection scheme, the detector is effectively biased by a current $I_{0}+\delta I(t)$. The switching of the detector junction out of the supercurrent state is detected as voltage pulses between points $A$ and $B$. The effects of non-zero higher odd moments of the distribution of $\delta I(t)$ can be probed by inverting the currents $-\bar{I}_{\mathrm{N}}$ and $\bar{I}_{\mathrm{N}}+\delta I(t)$, as indicated by the alternative signs in the figure. Inverting the average current through the noise source changes the sign of the third cumulant of the fluctuations, resulting in differing average escape rates of the detector.

$N$ current pulses to measure a single point on a histogram. The effects of noise at these low frequencies are considered for example in Ref. 25, and will not be discussed further in this work. Above this frequency range, weak current fluctuations with frequencies up to the order of $\omega_{\mathrm{p}}$ belong to the socalled adiabatic regime. These quasistationary fluctuations have low enough 


\section{Detecting Current Noise with a Josephson Junction}

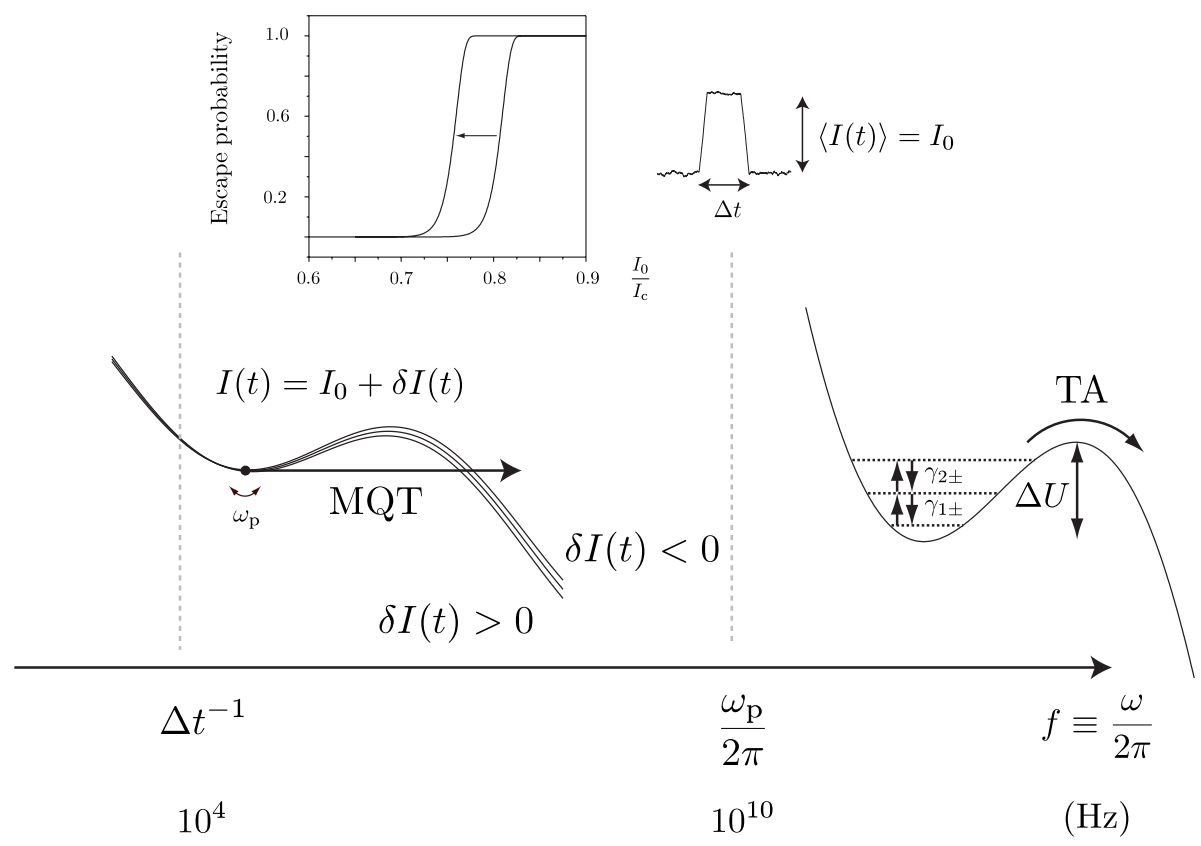

Figure 3. Typical behavior of a JJ in the presence of current fluctuations belonging to different frequency ranges as discussed in the text. The fluctuations $\delta I(t)$ cause the measured escape histograms to shift toward lower values of $I_{0}$. In this work we focus on the wide adiabatic regime spanning frequencies $f$ satisfying $\Delta t^{-1} \ll f \ll \frac{\omega_{\mathrm{p}}}{2 \pi}$. Here $\Delta t^{-1}$ is the inverse length of a bias current pulse and $\omega_{\mathrm{p}}$ is the plasma frequency of the detector junction. Current fluctuations in this regime slowly vary the tilt of the potential, and the escape rate is obtained as an average of the MQT rate over the distribution of the current values. On the other hand, high frequency noise with $f \sim \frac{\omega_{\mathrm{p}}}{2 \pi}$ excites the JJ from its ground state, leading to thermal activation at an effective temperature.

frequencies so that the noise $\delta I(t)$ can be considered merely as a parameter slowly varying the tilt of the washboard potential around its average. $\frac{26}{6}$ In this limit the junction remains in its instantaneous metastable quantum mechanical ground state that decays via MQT. Since the tunneling rate is strongly dependent on current, the junction can act as a detector of fluctuations.

Contrary to this weak low frequency noise, stronger fluctuations with frequencies comparable to $\omega_{\mathrm{p}}$ and thus to the level separation in the potential well excite the phase particle to higher energy levels, leading to escape over the barrier top. Again, the junction is sensitive to the fluctuations but this time the noise affects the rate of effective thermal activation instead of 


\section{J.T. Peltonen et al.}

quantum tunneling.

Although detecting the influence of the third cumulant of adiabatic current fluctuations is the primary goal of the measurement scheme considered in this work, we will first focus on the opposite, high-frequency limit. This is necessary as the power of noise at these high frequencies sets a condition for the validity of the discussion in the adiabatic regime.

\section{EFFECTS OF CURRENT FLUCTUATIONS AT NON-ADIABATIC FREQUENCIES}

To understand the influence of high-frequency noise on a JJ detector, let us briefly review the model introduced in Ref. 13. The effects of the second moment at high frequencies can be modeled as resonant excitation between different energy levels in the nearly harmonic potential well of the detector junction. At low temperatures, this process is mainly driven by the nonequilibrium shot noise. The transitions take place approximately at the plasma frequency of the junction, and the resulting level dynamics can be described in terms of an effective temperature $T^{*}$ leading to thermal activation over the potential barrier. In the following, we choose to denote the excitation and relaxation rates between the $j-1$ :th and $j$ :th level in the well separated by energy $\hbar \omega_{j, j-1}$ by $\gamma_{j, j-1}$ and $\gamma_{j-1, j}$, respectively.

To derive expressions for the transition rates, we start from the Hamiltonian $\mathcal{H}$ of a JJ biased with a fluctuating current $I(t)=I_{0}+\delta I(t)$ and write $\mathcal{H}$ in the general form as $\mathcal{H}(t)=\mathcal{H}_{0}+V(t)$, where $\mathcal{H}_{0}$ is the time-independent system Hamiltonian and $V(t)$ is a perturbation of the form $g \hat{A} \hat{f}(t)$. Here

$g$ is a coupling constant and $\hat{A}$ is an operator of the system (the detector junction). The matrix elements $\langle j|\hat{A}(t=0)| j-1\rangle$ of $\hat{A}$ between states $|j\rangle$ and $|j-1\rangle$ at $t=0$ are expressed as $A^{j, j-1}$. Further, $\hat{f}(t)$ is a fluctuating operator commuting with $\hat{A}$ with a power spectrum $S_{f}(\omega)$.

We proceed by expanding the unitary time-evolution of a state $\left|\psi_{I}(t)\right\rangle$ in the interaction picture up to third order in the perturbation $V(t)$ :

$$
\begin{aligned}
\left|\psi_{I}(t)\right\rangle \simeq & \left\{1-\frac{i}{\hbar} \int_{0}^{t} \mathrm{~d} \tau_{1} V\left(\tau_{1}\right)-\frac{1}{2 \hbar^{2}} \int_{0}^{t} \int_{0}^{t} \mathrm{~d} \tau_{1} \mathrm{~d} \tau_{2} \mathcal{T}\left[V\left(\tau_{1}\right) V\left(\tau_{2}\right)\right]\right. \\
& \left.+\frac{i}{6 \hbar^{3}} \int_{0}^{t} \int_{0}^{t} \int_{0}^{t} \mathrm{~d} \tau_{1} \mathrm{~d} \tau_{2} \mathrm{~d} \tau_{3} \mathcal{T}\left[V\left(\tau_{1}\right) V\left(\tau_{2}\right) V\left(\tau_{3}\right)\right]\right\}\left|\psi_{I}(0)\right\rangle,
\end{aligned}
$$

where the time-ordering operator $\mathcal{T}$ is needed as the operators $V(\tau)$ at different times do not necessarily commute. To study the influence of the second moment of fluctuations, we neglect temporarily the last two terms on the right hand side of Eq. (8). Now, considering the time-evolution of the ini- 


\section{Detecting Current Noise with a Josephson Junction}

tial state $\left|\psi_{I}(0)\right\rangle=|j\rangle$, we first find the probability amplitude $\alpha_{j-1, j}(t) \equiv$ $\langle j-1 \mid j(t)\rangle$ for a transition to state $|j-1\rangle$. The corresponding ensembleaveraged relaxation rate follows then from the standard expression 27

$$
\gamma_{j-1, j} \equiv \frac{\mathrm{d}}{\mathrm{d} t}\left\langle\left|\alpha_{j-1, j}(t)\right|^{2}\right\rangle=\frac{g^{2}}{\hbar^{2}}\left|A^{j, j-1}\right|^{2} S_{f}\left(\omega_{j, j-1}\right) .
$$

A similar calculation gives the excitation rate from the $j-1$ :th to the $j$ :th level as

$$
\gamma_{j, j-1} \equiv \frac{\mathrm{d}}{\mathrm{d} t}\left\langle\left|\alpha_{j, j-1}(t)\right|^{2}\right\rangle=\frac{g^{2}}{\hbar^{2}}\left|A^{j, j-1}\right|^{2} S_{f}\left(-\omega_{j, j-1}\right),
$$

implying that relaxation is mainly determined by the noise power at positive and excitation at negative frequencies.

The detector junctions usually have $E_{J} \gg E_{C}$, and we can write the Hamiltonian $\mathcal{H}_{0}$ in the tight-binding approximation of Eq. (2) with $I=I_{0}$ and further approximate the potential by a harmonic one. Referring to Eq. (2), we have $g=-E_{J} / I_{c}, \hat{A}=\varphi$ and $\hat{f}(t)=\delta I(t)$. The required matrix elements for the harmonic potential are given by $j / 2 \hbar \omega_{\mathrm{p}} C$, yielding $\gamma_{j-1, j} \simeq$ $\left(j / 2 \hbar \omega_{p} C\right) S_{I}\left(\omega_{j, j-1}\right)$ for the relaxation and $\gamma_{j, j-1} \simeq\left(j / 2 \hbar \omega_{p} C\right) S_{I}\left(-\omega_{j, j-1}\right)$ for the excitation rate. Here, the total noise power at the detector

$$
\begin{aligned}
S_{I}\left( \pm \omega_{j, j-1}\right) & =2 e F_{2} \bar{I}_{N} \alpha \operatorname{coth} \frac{e V}{2 k_{\mathrm{B}} T} \\
& +2 \hbar \omega_{j, j-1} \operatorname{Re}\left[Y\left(\omega_{j, j-1}\right)\right]\left(\operatorname{coth} \frac{\hbar \omega_{j, j-1}}{2 k_{\mathrm{B}} T} \pm 1\right)
\end{aligned}
$$

is a sum of the contributions from the shot noise source and the equilibrium fluctuations arising from the dissipative circuit surrounding the junction. The former is scaled by a factor $\alpha$ : It relates the noise power at the source to the noise power at the detector at frequency $\omega_{j, j-1}$. The frequencydependent factor $\alpha$ can be calculated by solving the set of Langevin equations written for each branch of the circuit, $\frac{17}{b}$ but for the present case we may combine it with $F_{2}$ into an effective Fano factor $F$ of the complete circuit. With this in mind, the transition rates read

$$
\begin{aligned}
\gamma_{j-1, j} & \simeq \frac{j F e \bar{I}_{\mathrm{N}} \operatorname{coth}\left(e V / 2 k_{\mathrm{B}} T\right)}{\hbar \omega_{\mathrm{p}} C}+j \frac{\omega_{j, j-1}}{Q}\left(\operatorname{coth} \frac{\hbar \omega_{j, j-1}}{2 k_{\mathrm{B}} T}+1\right) \\
\gamma_{j, j-1} & \simeq \frac{j F e \bar{I}_{\mathrm{N}} \operatorname{coth}\left(e V / 2 k_{\mathrm{B}} T\right)}{\hbar \omega_{\mathrm{p}} C}+j \frac{\omega_{j, j-1}}{Q}\left(\operatorname{coth} \frac{\hbar \omega_{j, j-1}}{2 k_{\mathrm{B}} T}-1\right) .
\end{aligned}
$$

Here, $Q \equiv C \omega_{\mathrm{p}} / \operatorname{Re}\left[Y\left(\omega_{j, j-1}\right)\right]$ denotes the quality factor of the detector junction. The level dynamics following from Eq. (12) is equivalent to that 


\section{J.T. Peltonen et al.}

arising from pure equilibrium fluctuations at an effective temperature $T^{*}$ if we require

$$
\begin{aligned}
\gamma_{j-1, j} & \equiv \frac{j \omega_{j, j-1}}{Q}\left(\operatorname{coth} \frac{\hbar \omega_{j, j-1}}{2 k_{\mathrm{B}} T^{*}}+1\right) \\
\text { and } \gamma_{j, j-1} & \equiv \frac{j \omega_{j, j-1}}{Q}\left(\operatorname{coth} \frac{\hbar \omega_{j, j-1}}{2 k_{\mathrm{B}} T^{*}}-1\right) .
\end{aligned}
$$

Comparing this expression with Eq. (12), we have, assuming $\omega_{j, j-1} \simeq \omega_{p}$,

$$
k_{\mathrm{B}} T^{*} \simeq \frac{\hbar \omega_{\mathrm{p}}}{2 \operatorname{arcoth}\left(\operatorname{coth} \frac{\hbar \omega_{\mathrm{p}}}{2 k_{\mathrm{B}} T}+\frac{Q F e \bar{I}_{\mathrm{N}} \operatorname{coth}\left(e V / 2 k_{\mathrm{B}} T\right)}{\hbar \omega_{\mathrm{p}}^{2} C}\right)} .
$$

In the absence of shot noise when $\bar{I}_{\mathrm{N}}=0, T^{*}$ reduces to the actual temperature $T$. For bias voltages $e|V| \gg k_{\mathrm{B}} T$ together with $\hbar \omega_{\mathrm{p}} \gg k_{\mathrm{B}} T, T^{*}$ is obtained from

$$
k_{\mathrm{B}} T^{*} \simeq \frac{\hbar \omega_{p}}{2 \operatorname{arcoth}\left(1+\frac{Q F e\left|\bar{I}_{\mathrm{N}}\right|}{\hbar \omega_{p}^{2} C}\right)} .
$$

Consequently, for high noise currents with $\left|\bar{I}_{\mathrm{N}}\right| \gg \hbar \omega_{\mathrm{p}}^{2} C / Q F e$, Eq. (15) gives $T^{*} \simeq Q F e\left|\bar{I}_{\mathrm{N}}\right| / 2 k_{\mathrm{B}} \omega_{\mathrm{p}} C=F e\left|\bar{I}_{\mathrm{N}}\right| / 2 \operatorname{Re}\left[Y\left(\omega_{\mathrm{p}}\right)\right]$. On the other hand, if the noise currents are still in the high limit but $\hbar \omega_{\mathrm{p}} \lesssim 2 k_{\mathrm{B}} T$, the effective temperature contains also a term proportional to temperature $T$ :

$$
k_{\mathrm{B}} T^{*} \simeq k_{\mathrm{B}} T+\frac{Q F e\left|\bar{I}_{\mathrm{N}}\right|}{2 \omega_{\mathrm{p}} C}
$$

A similarly defined effective temperature is employed also in Ref. 16.

Now, if $T^{*}$ exceeds the crossover temperature for quantum tunneling, i.e., $T^{*}>T_{0} \equiv \hbar \omega_{p} / 2 \pi k_{\mathrm{B}}$, the decay of the metastable state occurs primarily via thermal activation over the potential barrier ${ }^{24}$ Assuming a bias current $I_{0}$ close to $I_{\mathrm{c}}$, the potential can again be approximated by a cubic parabola and the escape rate $\Gamma$ is obtained from (7) with $T=T^{*}$. The switching probability of the junction for a bias current pulse of height $I_{0}$ and length $\Delta t$ is correspondingly given by

$$
P\left(I_{0}\right)=1-\exp \left(-\Gamma\left(I_{0}\right) \Delta t\right)
$$

allowing comparison with experimental switching histograms at different values of $\bar{I}_{\mathrm{N}}$. For example, the $I_{0}$ values corresponding to $50 \%$ escape probability, $P\left(I_{0}\right)=0.5$, or the difference in $I_{0}$ between the $10 \%$ and $90 \%$ points allow for a straightforward comparison of the theoretical model with experimental data. 


\section{Detecting Current Noise with a Josephson Junction}

Besides describing the influence of non-adiabatic fluctuations, the effective temperature sets a limit for the applicability of the adiabatic model. To find the noise current $\left|\bar{I}_{N, 0}\right|$ corresponding to the crossover temperature $T_{0}$ we require that

$$
1+\frac{Q F e\left|\bar{I}_{\mathrm{N}, 0}\right|}{\hbar \omega_{\mathrm{p}}^{2} C}=\operatorname{coth} \pi
$$

by use of which we can estimate that the currents $\left|\bar{I}_{\mathrm{N}}\right|$ have to stay considerably below

$$
\left|\bar{I}_{\mathrm{N}, 0}\right| \equiv \frac{\eta \hbar \omega_{\mathrm{p}}^{2} C}{Q F e}=\frac{2 \eta I_{\mathrm{c}} \varphi_{0}}{Q F}, \quad \eta \equiv \operatorname{coth} \pi-1 \sim 0.0037
$$

for MQT to be the main escape mechanism. This can be used as a first approximation of the limit of validity for an adiabatic description of the bias current fluctuations. Imposing such a limit is necessary since the effects of the third and higher moments are masked by the second-moment induced high frequency effects. To keep the currents $\bar{I}_{\mathrm{N}}$ in an experimentally reasonable regime, the factor $Q F \equiv F_{2} \alpha\left(\omega_{\mathrm{p}}\right) C \omega_{\mathrm{p}} / \operatorname{Re}\left[Y\left(\omega_{\mathrm{p}}\right)\right]$ in Eq. (19) can be adjusted by using a desired filtering circuit, as will be discussed in Sec. 6. As an example, let us consider a detector with $I_{\mathrm{c}}=5 \mu \mathrm{A}$ and $C=40$ $\mathrm{fF}$ which could well correspond to the parameters of the junction in Fig. 2, If the junction is biased at $I_{0}=0.5 I_{\mathrm{c}}$ and the limiting noise current $I_{\mathrm{N}, 0}$ is required to be $5 \mu \mathrm{A}$ for typical values $Q \simeq 10$ and $F_{2}=1$, the factor $\alpha\left(\omega_{\mathrm{p}}\right)$ related to the filtering should be of the order of $10^{-3}$.

\subsection{Higher order effects in the non-adiabatic frequency regime}

When deriving expressions for the above relaxation and excitation rates, we assumed a harmonic potential. The corrections to the transition rates arising from the anharmonicity of the cubic potential can be handled in perturbation theory. Moreover, the influence of higher cumulants of current fluctuations is revealed by taking into account more terms in the expansion of Eq. (8). Yet, in the previous switching measurements ${ }^{13}$ these non-Gaussian features were masked by the effects of the second moment, well described by the above model with an effective temperature. Other experimental detection schemes considering JJs as detectors of high frequency fluctuations may thus become more suitable in the non-adiabatic frequency regime when focusing on the higher order effects.

The weakness of the third-order effects is evident if we consider all the terms in Eq. (8) and in addition account for the anharmonicity of the cubic 


\section{J.T. Peltonen et al.}

potential in first-order perturbation theory. Using the scattering matrixbased in-out ordering $\frac{19}{19}$ of the current operators, the averaged time-ordered products of the operators can be expressed in terms of three-current correlators or their spectral functions analogous to the noise power. Assuming these functions to be frequency-independent for the range of relevant frequencies, we find in the zero-temperature limit that the shot-noise induced transition rate from the initial $j$ :th state to the final $j+1$ :th state is given by

$$
\gamma_{j+1, j}=(j+1) \frac{F e\left|\bar{I}_{\mathrm{N}}\right|}{\hbar \omega_{\mathrm{p}} C} .
$$

This is nothing but the first term in Eq. (12), arising from a second order contribution in a harmonic potential - the third order contribution vanishes identically. On the other hand, an initial superposition state like $\kappa_{j}|j\rangle+$ $\kappa_{j+1}|j+1\rangle$ leads to oscillating populations of the different energy levels: The frequency of these oscillations corresponds to the level separation $\omega_{01}$, whereas their amplitude is proportional to the third moment of the noise source. For a noise source with Fano factor $F_{3}$, a part of this third order contribution originates from a term $\left\langle j+1\left|\mathcal{T}\left[V\left(\tau_{1}\right) V\left(\tau_{2}\right) V\left(\tau_{3}\right)\right]\right| j\right\rangle$. This contributes to the occupation probability of the $j+1$ :th level by

$$
\begin{aligned}
& \int_{0}^{t} \int_{0}^{t} \int_{0}^{t} \mathrm{~d} \tau_{1} \mathrm{~d} \tau_{2} \mathrm{~d} \tau_{3}\left\langle j+1\left|\mathcal{T}\left[V\left(\tau_{1}\right) V\left(\tau_{2}\right) V\left(\tau_{3}\right)\right]\right| j\right\rangle \\
& =F_{3} e^{2} \alpha_{0}^{3} \frac{(j+1)^{3 / 2}}{\hbar^{3} \omega_{01}} \bar{I}_{\mathrm{N}}\left[1-\cos \left(\omega_{01} t\right)\right],
\end{aligned}
$$

where $\alpha_{0} \equiv \frac{\hbar}{2 e}\left(\frac{2 E_{\mathrm{C}}}{E_{\mathrm{J}}}\right)^{1 / 4}$. Correspondingly, the average transition rate arising from this term vanishes, predicting that there should be no detectable net signal from the third order resonant transitions in the switching measurement scheme discussed above.

Considering the other recently proposed approaches to detecting higherorder cumulants, Ankerhold ${ }^{16}$ has used an effective Fokker-Planck equation to describe the influence of weak short-correlated non-Gaussian fluctuations on a Josephson junction in the regime of thermal activation. The detection is again based on the sensitivity of the switching rate out of the zero-voltage state to variations in current, and the principle of Fig. 2 can be used to reveal the presence of higher odd cumulants.

Another approach has been to consider a noise source capacitively coupled to a small JJ in the regime of incoherent Cooper pair tunneling. third cumulant of high frequency current fluctuations should then be detectable by comparing the $I-V$-curves of the detector junction at different values of the noise current. On the other hand, in Ref. 28 the authors 


\section{Detecting Current Noise with a Josephson Junction}

investigate the influence of third moment of current fluctuations on a two level system. This detector can be experimentally realized as a hysteretic JJ SQUID. The master equation approach considers the time-evolution of the reduced density matrix of the two level system and predicts third-moment induced coherent oscillations between different states, observable for example by studying Rabi oscillations of the system. This result is consistent with Eq. (21), bearing in mind that Eq. (21) is obtained for a multilevel system, not a qubit. A similar master equation approach was employed also in Ref. 29 to calculate transition rates induced by the third cumulant.

Furthermore, a measurement with a mesoscopic conductor parallel to a current-biased JJ has been predicted to reveal the fourth cumulant of current fluctuations. ${ }^{14}$ This is based on a modification of the rate of macroscopic quantum tunneling by the mesoscopic conductor. The principle is somewhat similar to detecting the influence of the third cumulant in the regime of adiabatic fluctuations, as we will discuss below in more detail.

\section{INFLUENCE OF THE THIRD CUMULANT IN THE ADIABATIC FREQUENCY REGIME}

The above model with the effective temperature relied heavily on excitation and relaxation between different energy levels in the potential well of the detector junction. In this section we concentrate on fluctuations of lower frequencies that do not excite the detector to higher levels.

To analyze the adiabatic fluctuations quantitatively, let us first recall that the current distribution $\rho(\delta I)$ around the average current $I_{0}$ is obtained as the Fourier transform $\rho(\delta I)=\frac{1}{2 \pi} \int_{-\infty}^{\infty} \mathrm{d} k e^{-i k \delta I} \phi_{\delta I}(k)$ of the characteristic function $\phi_{\delta I}(k)$. With $c_{n}$ denoting the cumulants of the current, the char-

acteristic function can be expressed as $\phi_{\delta I}(k)=\exp \left(\sum_{n=2}^{\infty} \frac{(i k)^{n}}{n !} c_{n}\right)$. Since the fluctuations are centered around the average current, $\langle\delta I\rangle=c_{1}=0$. Consequently, the second and third cumulants are given by $c_{2}=\left\langle\delta I(t)^{2}\right\rangle$ and $c_{3}=\left\langle\delta I(t)^{3}\right\rangle$, and for simplicity we assume the distribution to be stationary. Further, assuming the fourth and higher cumulants to be negligibly small, we concentrate on the influence of the third cumulant. Truncating the sum in the characteristic function at $n=3$, one finds the corresponding approximation to the probability density as

$$
\rho(\delta I) \simeq \frac{1}{\sqrt{2 \pi c_{2}}}\left(1-\frac{c_{3}}{2 c_{2}^{2}} \delta I+\frac{c_{3}}{6 c_{2}^{3}} \delta I^{3}\right) \exp \left(-\frac{\delta I^{2}}{2 c_{2}}\right) .
$$

This result is valid when the skewness $\gamma$ of the current distribution is small, i.e., when $\gamma \equiv c_{3} / c_{2}^{3 / 2} \ll 1.30$ 


\section{J.T. Peltonen et al.}

Now, for a JJ biased by a current $I$ close to the critical current $I_{\mathrm{c}}$, the MQT rate $\Gamma(I)$ is given by Eq. (5). Writing the current as $I(t)=I_{0}+\delta I(t)$, the escape probability for a current pulse of height $I_{0}$ and length $\Delta t$ is obtained from

$$
P\left(I_{0}\right)=1-\exp \left(-\int_{0}^{\Delta t} \Gamma\left(I_{0}+\delta I(t)\right) \mathrm{d} t\right) .
$$

For adiabatic current fluctuations $\delta I(t)$ with frequencies above the inverse pulse length $\Delta t^{-1}$ but well below the plasma frequency $\omega_{\mathrm{p}} / 2 \pi$, we can assume the fluctuations to be ergodic. In this case the time average of the escape rate can be replaced by an ensemble average over the distribution of fluctuations, giving $\langle\Gamma\rangle$ as

$$
\langle\Gamma\rangle \simeq \int_{-\infty}^{\infty} d \delta I \rho(\delta I) \Gamma\left(I_{0}+\delta I\right)
$$

This corresponds further to an escape probability

$$
P\left(I_{0}\right)=1-\exp (-\langle\Gamma\rangle \Delta t)
$$

which can be directly compared with experimental escape histograms.

For data fitting we obtain values of $\langle\Gamma\rangle$ by numerical integration but for illustration we can write a second order approximation in $\delta I$ for $\Gamma\left(I_{0}+\delta I\right)=$ $\exp \left[\ln A\left(I_{0}+\delta I\right)-B\left(I_{0}+\delta I\right)\right]$, giving together with Eqs. (22) and (24) the result

$$
\langle\Gamma\rangle \simeq \frac{\Gamma_{0}}{\sqrt{\hat{B} c_{2}}} \exp \left(\frac{\hat{A}^{2}}{2 \hat{B}}\right)\left[1-\frac{c_{3}}{2 c_{2}^{2}} \frac{\hat{A}}{\hat{B}}+\frac{c_{3}}{6 c_{2}^{3}}\left(\frac{\hat{A}}{\hat{B}}\right)^{3}+\frac{c_{3}}{2 c_{2}^{3}} \frac{\hat{A}}{\hat{B}^{2}}\right] .
$$

Here $\Gamma_{0}$ is the unperturbed tunneling rate given by Eq. (5) with $I=I_{0}$ and we introduced the parameters

$$
\hat{A} \equiv \frac{\partial}{\partial I}[\ln A(I)-B(I)]_{I=I_{0}}
$$

and

$$
\hat{B} \equiv \frac{1}{c_{2}}-\frac{\partial^{2}}{\partial I^{2}}[\ln A(I)-B(I)]_{I=I_{0}} .
$$

This approximation is naturally valid only for small variations of current. Moreover, neglecting the current dependence of the prefactor $A$ and taking only first order corrections in the exponent $B(I)$ into account, Eq. (26) reduces to

$$
\langle\Gamma\rangle \simeq \Gamma_{0} \exp \left[\frac{1}{2}\left(\frac{\partial B}{\partial I}\right)^{2}\left\langle\delta I^{2}\right\rangle\right]\left[1-\frac{1}{6}\left(\frac{\partial B}{\partial I}\right)^{3}\left\langle\delta I^{3}\right\rangle\right] .
$$




\section{Detecting Current Noise with a Josephson Junction}

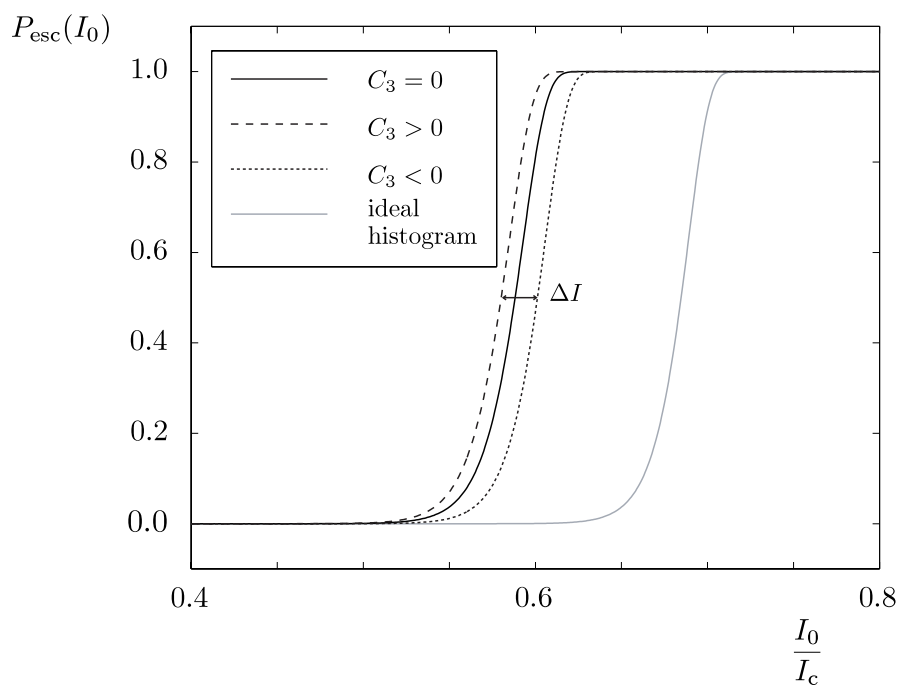

Figure 4. An example of the shift of the escape histograms compared to the ideal noiseless case when adiabatic fluctuations are present at the detector junction. The left group of three curves corresponds to a Gaussian noise source with $c_{3}=0$ and a non-Gaussian source with equal $c_{2}$ but $c_{3} \neq 0$. Additionally, as discussed below in more detail, the arrow indicates the shift $\Delta I$ in bias currents corresponding to a fixed threshold probability $(50 \%)$ for opposite signs of $c_{3}$. The detector is assumed to have $I_{\mathrm{c}}=1 \mu \mathrm{A}$ and $C=20$ $\mathrm{fF}$, whereas $c_{2}$ and $c_{3}$ are calculated for a current $\bar{I}_{\mathrm{N}}=0.5 \mu \mathrm{A}$ in an ideal circuit with a cutoff frequency at $0.5 \omega_{\mathrm{p}}$.

When the approximation of Eq. (22) is valid, we see that the main effect of a nonzero current $\bar{I}_{N}$ through the shot noise source is to change the average tunneling rate, resulting in a shift of the escape probability histogram. This is illustrated in Fig. 4 for a Gaussian source and two non-Gaussian sources with third cumulants of opposite signs. The current corresponding to a fixed switching probability is clearly reduced compared to the noiseless histogram. In Fig. 5 such current suppression is shown for the current corresponding to $50 \%$ switching probability when $\bar{I}_{\mathrm{N}}$ is varied.

For different polarities of $\bar{I}_{N}$, the change in $\langle\Gamma\rangle$ has the same magnitude but different direction. In first approximation, this effect is caused solely by the third cumulant. For numerical evaluation of the escape rate asymmetry 


\section{J.T. Peltonen et al.}

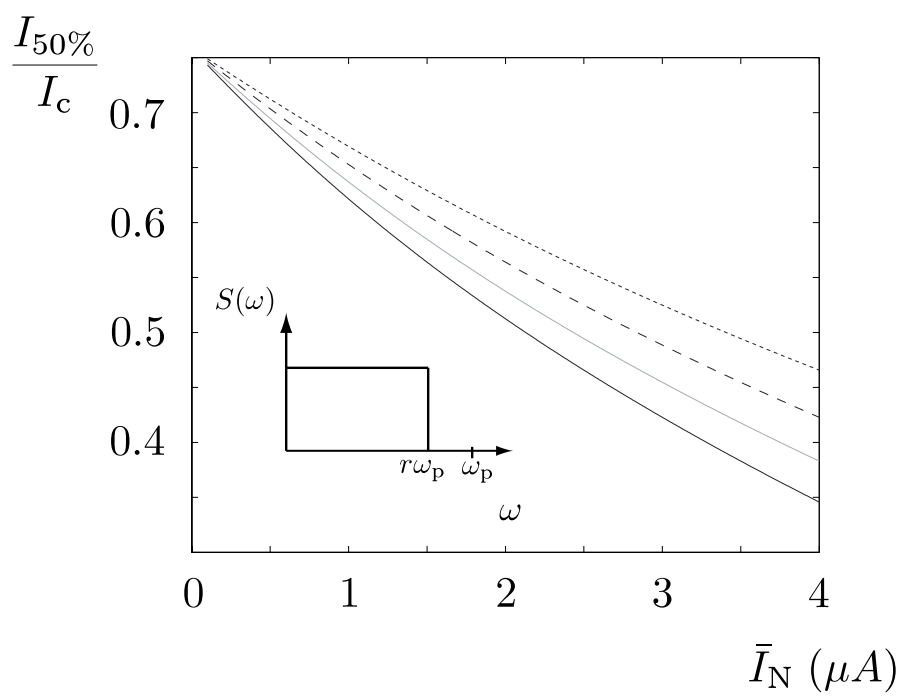

Figure 5. The bias current $I_{0}$ corresponding to a $50 \%$ switching probability as a function of $\bar{I}_{\mathrm{N}}$, the average current through the noise source with a third cumulant $c_{3}=0$ within the adiabatic model. The detector is assumed to have $I_{\mathrm{c}}=2 \mu \mathrm{A}$ and $C=20 \mathrm{fF}$. Furthermore, the circuit around the JJ is assumed to have a flat frequency response up to a cutoff at $r \omega_{\mathrm{p}} / 2 \pi$, as shown in the inset. The curves from top to bottom correspond to different values of $r$ between 0.5 and 0.8 in steps of 0.1 .

between different polarities of $\bar{I}_{N}$, we obtain

$$
\begin{aligned}
\langle\Gamma\rangle_{ \pm} & =\frac{1}{\sqrt{2 \pi}} \int_{-\infty}^{\infty} d x \Gamma_{0}\left(I_{0}+\sqrt{c_{2}} x\right) \exp \left(-x^{2} / 2\right) \\
& \pm \frac{\gamma}{2} \frac{1}{\sqrt{2 \pi}} \int_{-\infty}^{\infty} d x \Gamma_{0}\left(I_{0}+\sqrt{c_{2}} x\right) x\left(1-x^{2} / 3\right) \exp \left(-x^{2} / 2\right),
\end{aligned}
$$

which follows from Eqs. (22) and (24). This can then be used to evaluate the relative asymmetry of $\langle\Gamma\rangle$ between different polarities of $\bar{I}_{N}$, defined by the expression

$$
\frac{\Delta \Gamma}{\Gamma_{\mathrm{ave}}} \equiv \frac{\langle\Gamma\rangle_{+}-\langle\Gamma\rangle_{-}}{\frac{1}{2}\left(\langle\Gamma\rangle_{+}+\langle\Gamma\rangle_{-}\right)}
$$

The above result can further be used when approximating how a certain point on the histogram corresponding to a fixed switching probability moves as the sign of $\bar{I}_{\mathrm{N}}$ is changed. This shift $\Delta I$ of a threshold current $I_{0}$ is illustrated in Fig. 6. The value of $\Delta I$ is numerically easily obtained, e.g., by using a bisection method. In the linear approximation, this shift due to the third moment is determined by the combined effect of the asymmetry 


\section{Detecting Current Noise with a Josephson Junction}

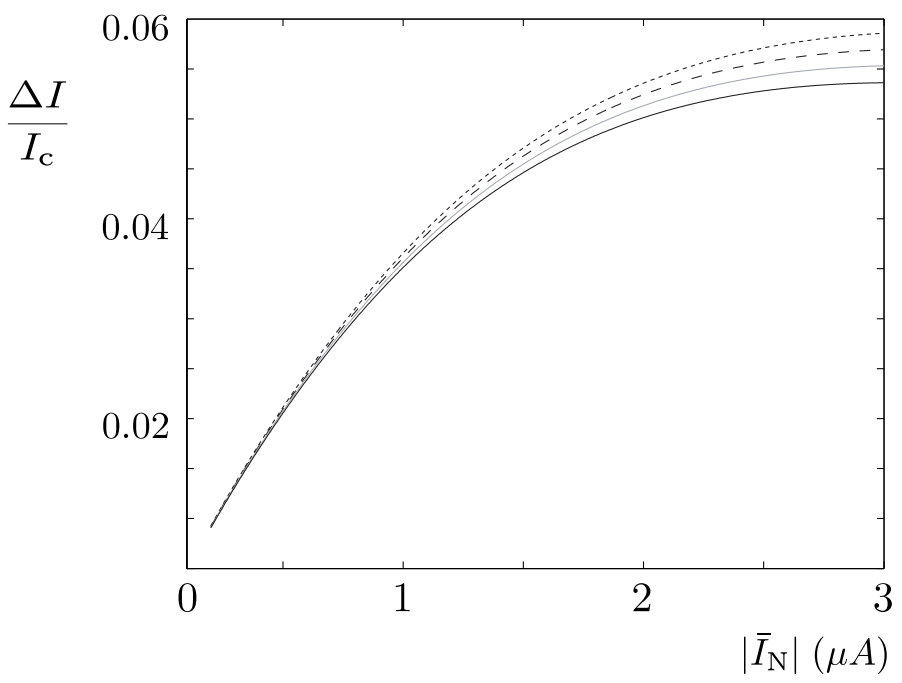

Figure 6. The shift $\Delta I / I_{\mathrm{c}}$ of a threshold current $I_{x}$ as a function of $\left|\bar{I}_{\mathrm{N}}\right|$, the magnitude of the average current through the noise source. $\Delta I$ is the difference of the bias currents $I_{0}$ corresponding to an escape probability $P=x$ when the polarity of $\bar{I}_{\mathrm{N}}$ is changed. The different curves from top to bottom correspond to values of $x$ between 0.5 and 0.8 in steps of 0.1 . The detector is again assumed to have $I_{\mathrm{c}}=1 \mu \mathrm{A}$ and $C=20 \mathrm{fF}$. In addition, the circuit around the JJ has a flat response up to a cutoff at $r \omega_{\mathrm{p}} / 2 \pi$ with $r=0.5$.

described by Eq. (31) and the slope of the histogram at the average current $I_{0}, \partial P /\left.\partial I\right|_{I=I_{0}}$. Thus, for $\Delta I \ll$ the width of the histogram, we find

$$
\Delta I \simeq(1-P) \ln (1-P)\left(\frac{\partial P}{\partial I}\right)^{-1} \frac{\Delta \Gamma}{\Gamma_{\text {ave }}}
$$

for the shift in the threshold current $I_{0}$ as $\bar{I}_{N}$ is changed to $-\bar{I}_{N}$.

\section{EXPERIMENTAL REALIZATION}

In Sec. 2.2. and in Fig. 2 we briefly described the principle of detecting current fluctuations using a Josephson junction. In this section we aim to give a more detailed view, focusing on the electrical circuit model illustrated in Fig. 7 .

The circuit is generally fabricated on a silicon substrate using standard electron beam lithography and shadow evaporation of aluminium. For measurements the circuit is cooled in a dilution refrigerator down to low 


\section{J.T. Peltonen et al.}

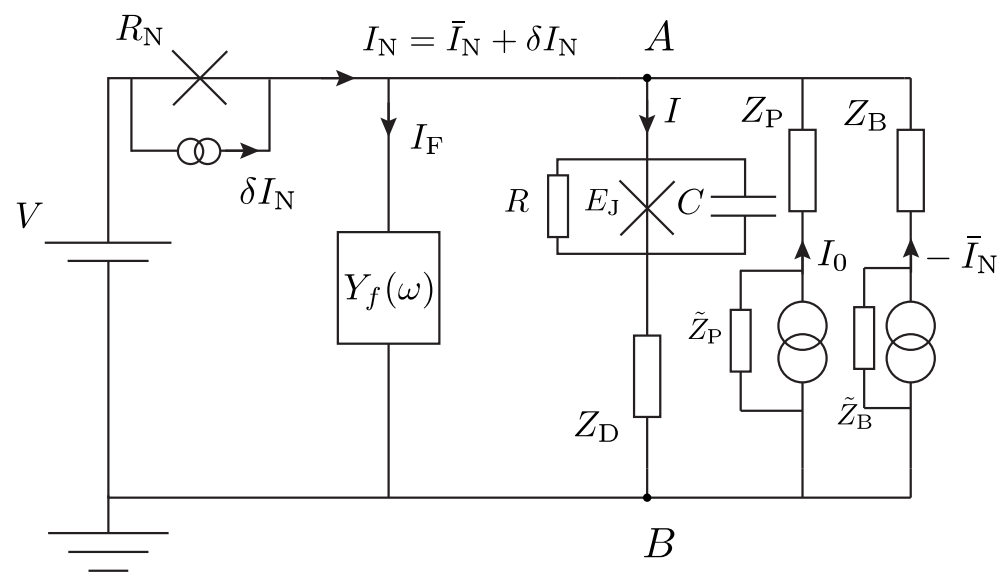

Figure 7. Circuit model for the electrical environment of the JJ used as a noise detector. The impedances $Z_{\mathrm{D}}, Z_{\mathrm{P}}$ and $Z_{\mathrm{B}}$ are mainly inductive whereas $\tilde{Z}_{\mathrm{P}}$ and $\tilde{Z}_{\mathrm{B}}$ are capacitive. The bias currents $I_{0}$ and $-\bar{I}_{\mathrm{N}}$ are realized by applying a bias voltage over a large resistor. When the detecting junction switches to the normal state, voltage pulses are measured, e.g., between points $A$ and $B$.

temperatures $(T<50 \mathrm{mK})$, where MQT is the dominating escape mechanism in the detector, as desired. The shot noise source is typically another small superconducting tunnel junction in the normal state biased by a constant voltage $V$. In practise, the noise source is typically realized as another Josephson junction with a critical current much smaller than that of the detector junction. Referring to Fig. 2, the average current through this mesoscopic scatterer is given by $\bar{I}_{\mathrm{N}}=V / R_{\mathrm{N}}$, where $R_{\mathrm{N}}$ is the resistance of the junction at the bias voltage $V$. For convenience, we separate the DC and AC parts of the current $I_{\mathrm{N}}$ as $I_{\mathrm{N}}=\bar{I}_{\mathrm{N}}+\delta I_{\mathrm{N}}$. Without extra considerations, a part of or all of the DC current $\bar{I}_{\mathrm{N}}$ will flow through the detector. Therefore, in a typical measurement this DC component of the current flowing through the scatterer junction is balanced by applying a constant current $-\bar{I}_{\mathrm{N}}$ through an inductive line. This current bias is realized by applying a voltage over a large resistance at room temperature $(R \geq 1 \mathrm{M} \Omega)$. As a result, no DC current flows through the detector junction until a current pulse $I_{0}$ is injected through a similarly prepared line as for the balancing current $-\bar{I}_{\mathrm{N}}$. The above procedure allows a more accurate detection of the fluctuations for different directions of $\bar{I}_{\mathrm{N}}$, and the balance of DC currents is adjusted and monitored for each value of $V$ ( or $\bar{I}_{\mathrm{N}}$ ).

After the circuit has been balanced, a current pulse $I_{0}$ is applied. The current $I_{0}$ has the form of a trapezoidal pulse with height $I_{0}$. The pulse has 


\section{Detecting Current Noise with a Josephson Junction}

long leading and trailing edges to ensure that the detector responds adiabatically to the changing bias current. Typical pulse lengths vary between 100 $\mu \mathrm{s}$ and $10 \mathrm{~ms}$, whereby applying a pulse corresponds to biasing the detector with a constant current $I_{0}$. Most importantly, the fluctuations caused by the shot noise source and possibly attenuated by a low-pass filter pass primarily through the detector because of the inductive filtering $\left(Z_{\mathrm{P}}\right.$ and $\left.Z_{\mathrm{B}}\right)$ of the lines with the current sources. These fluctuations imposed on top of the constant bias $I_{0}$ can then be probed by sending a large number $N$ (typically $>1000$ ) of bias pulses of the above kind at a fixed value of $I_{0}$ repeatedly through the detector to produce escape histograms.

The filter circuit with admittance $Y_{f}(\omega)$ in Fig. 7 is essential for guaranteeing the adiabaticity of the current fluctuations, as we will discuss in the next section. However, it does not qualitatively affect the measurement process described above. On the other hand, the effective admittance $Y(\omega)$ parallel to the detector junction gives an additional contribution to the tunneling exponent and prefactor of Eq. (5). To first order, the change of the tunneling exponent $B$ can be taken into account for arbitrary $Y(\omega)$ following Refs. 31 and 32, This results in a correction term $\Delta B$, which is, however, independent of the bias current through the junction. Moreover, the electrical circuit around the detector affects the cumulants $c_{2}$ and $c_{3}$ appearing in Eq. (22). These are related to the bias current fluctuations over the detector $\Delta I_{\mathrm{D}}$ and their distribution, which generally differs from that of the fluctuations $\delta I_{\mathrm{N}}$ at the noise source.

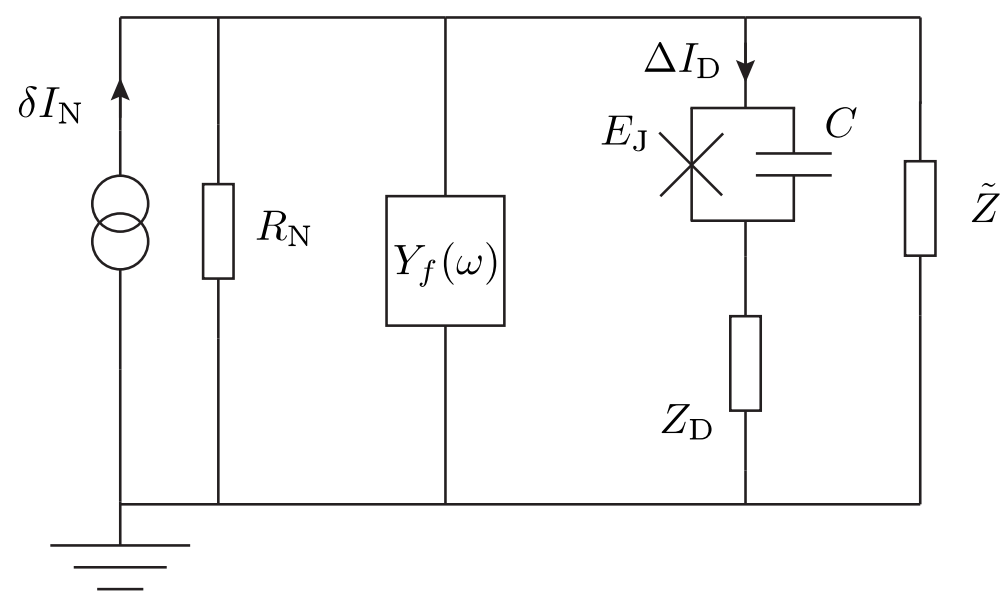

Figure 8. The circuit of Fig. 7 at AC frequencies. The impedance $\tilde{Z}$ represents the biasing and balancing circuit with the DC sources removed. The fluctuating current $\delta I_{\mathrm{N}}$ can be related to $\Delta I_{\mathrm{D}}$ by solving the set of Langevin equations as explained in the text. 


\section{J.T. Peltonen et al.}

At AC currents, the circuit appears as in Fig. 8. Modeling the noise source as a current generator with current $\delta I_{\mathrm{N}}$ parallel with a resistance $R_{\mathrm{N}}$, we have to relate the varying current $\Delta I_{\mathrm{D}}$ through the detector back to $\delta I_{\mathrm{N}}$. After this, the minimal-correlation estimates for the cumulants can be calculated by multiplication and subsequent integration over the relevant frequency interval. To achieve this, the current $\Delta I_{\mathrm{D}}$ is expressed in terms of $\delta I_{\mathrm{N}}$ by solving the set of Langevin equations of the circuit 17 : For each branch $j$, we first write the total current fluctuation as $\Delta I_{j}(\omega)=\Delta V_{j}(\omega) Y_{j}(\omega)+$ $\delta I_{j}(\omega)$. Here $Y_{j}(\omega)$ is the admittance of the single circuit element in branch $j$. This separates the contributions of a fluctuating potential drop $\Delta V_{j}(\omega)$ and those of an actual noise current source, $\delta I_{j}(\omega)$. For the circuit of Fig.

8, the only non-zero $\delta I_{j}(\omega)$-term corresponds naturally to $\delta I_{\mathrm{N}}$. The set of these Langevin equations can then be written as a matrix equation, whose solution gives for example the desired relation between $\Delta I_{\mathrm{D}}$ and $\delta I_{\mathrm{N}}$. This procedure corresponds to calculating the factor $\alpha$ appearing in the effective temperature model discussed in Sec. 3. Furthermore, the relations thus obtained can be used to study additional corrections to the third moment arising from the electrical circuit and the second moment of fluctuations. ${ }^{3314}$

\section{FILTERING REQUIREMENTS FOR THE MEASUREMENT OF ADIABATIC FLUCTUATIONS}

Above we have analyzed the effects on the probability histograms arising from the third cumulant of current fluctuations. Adiabaticity of the fluctuations is an essential requirement for the theoretical model of Sec. 4. to be applicable at all. Low temperature ensures thermal excitation to be negligible so that the detector junction stays in its metastable ground state in the absence of nonequilibrium fluctuations. On the other hand, in the presence of a shot noise source we have to ensure that the electrical circuit surrounding the detector junction behaves in a desirable way at high frequencies. If the noise power is considerable at frequencies near $\omega_{\mathrm{p}}$ we cannot apply the adiabatic model.

To use the adiabatic model over a wider range of $\bar{I}_{\mathrm{N}}$, a filtering circuit has to be engineered to suppress the high frequency nonequilibrium fluctuations. This is illustrated in Fig. 7 by the filter described with a frequency-dependent admittance $Y_{f}(\omega)$. Ideally, the frequency response of the filter and the rest of the circuit surrounding the detector, i.e., the total effective admittance $Y(\omega)$, should resemble that of a low-pass filter with a sharp cut-off below $\omega_{\mathrm{p}}$. Due to the admittance $Y(\omega)$, the spectral density of fluctuations at the detector should behave ideally as in Fig. 9, Since $\omega_{\mathrm{p}}$ 


\section{Detecting Current Noise with a Josephson Junction}

lies generally in the range of $10-100 \mathrm{GHz}$ and the filter circuit has to cover effectively a large bandwidth, the high frequencies require us to model the circuit using techniques of microwave engineering instead of relying merely on a lumped element analysis.

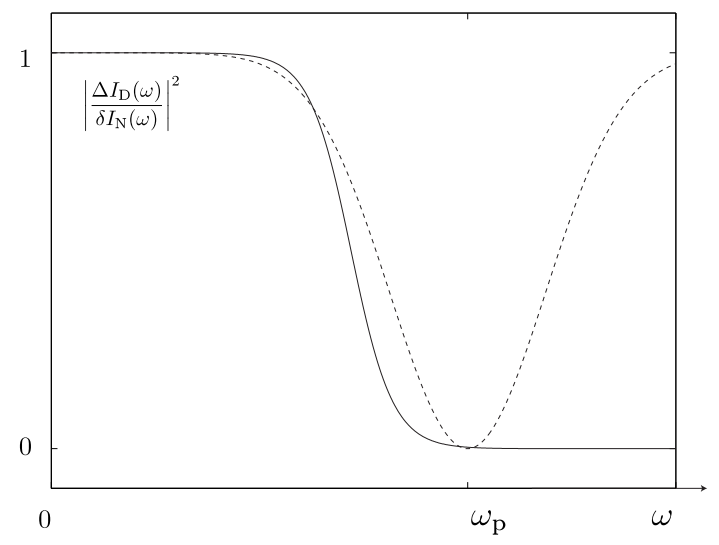

Figure 9. Idealized frequency responses of the on-chip filtering circuit in case of a low-pass filter (solid) or a band-stop filter (dashed). The vertical scale corresponds to the squared ratio of the current fluctuation $\Delta I_{\mathrm{D}}(\omega)$ at the detector junction to the current fluctuation $\delta I_{\mathrm{N}}(\omega)$ at the noise source, as illustrated in Fig. 8. The characteristics are adjusted so that fluctuations at the plasma frequency are suppressed by a desired amount.

In practise, a band reject filter with a notch around $\omega_{\mathrm{p}}$ is easier to implement than a good low-pass filter. This frequency response will suppress partially the transitions caused by the second moment provided the rejection band $\Delta \omega$ is wider than the plasma-resonance, $\Delta \omega>\omega_{\mathrm{p}} / Q$. Moreover, if the detector junction is replaced by a low-inductance DC SQUID with a tunable plasma frequency, performing noise spectroscopy of the shot noise source or characterization of the filter circuit becomes possible.

Considering the experimental realization of such a filter, we manufactured a sample containing a JJ acting as the noise source and a JJ detector separated from the source by a band-stop filter with a stopband centered around 30-40 GHz. The theoretical frequency response of the circuit is shown as the solid line in Fig. 10 b), whereas Fig. 10 a) shows experimental results of the current $I_{0.5}$ corresponding to $50 \%$ switching probability as a function of $\left|\bar{I}_{\mathrm{N}}\right|$ measured using the principle of Fig. 2. The detector is a DC SQUID with two parallel junctions with a maximum critical current of $3.7 \mu \mathrm{A}$ and a total capacitance of $100 \mathrm{fF}$. Likewise, a JJ with critical current $I_{\mathrm{c}, \mathrm{scat}} \simeq 1.6$ $\mu \mathrm{A}$ acts as the noise source. The different curves correspond to different 


\section{J.T. Peltonen et al.}
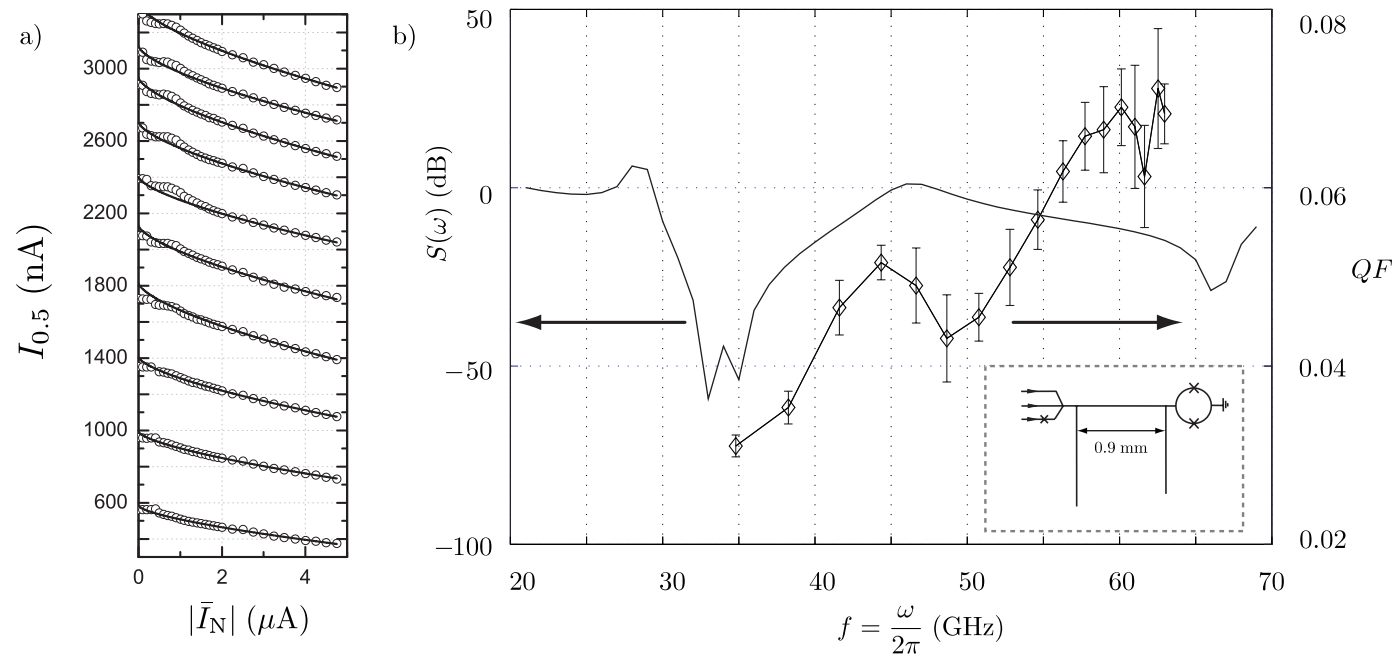

Figure 10. a) Measured $I_{0.5}$ values (open circles) as a function of $\left|\bar{I}_{\mathrm{N}}\right|$ together with theoretical curves calculated using the effective temperature mode ${ }^{13}$ with $Q F$ as a fitting parameter. Different curves correspond to different values of magnetic flux through the SQUID loop. The experimental points below $1 \mu \mathrm{A}$ lie almost at a constant level because the noise source is in the superconducting state. b) The $Q F$-values obtained from the fits in a) as a function of $\omega_{\mathrm{p}}$ (right scale). The factor $Q F$ is partially determined by the frequency response of the on-chip filtering circuit, the theoretical behavior of which is shown as the solid line (left scale). The inset shows a schematic of the sample (cf. Fig. 2), in which the junction acting as a noise source and the detector SQUID are separated by a band-stop filter.

values of magnetic flux through the SQUID loop, and therefore to different effective values of $I_{\mathrm{c}}$ and $\omega_{\mathrm{p}}$ of the detector. For this particular sample, the quality factor was very low, $Q \sim 2$, whereby the energy levels are not well separated. A relation between $I_{0.5}$ and $\left|\bar{I}_{\mathrm{N}}\right|$ can be obtained using Eqs. (77) and (6) together with $T^{*}$ from (15), as discussed in Ref. 13. With the factor $Q F$ as a fitting parameter, one obtains the solid lines in Fig. 10 a). Consequently, Fig. [10 b) shows the variation of these $Q F$-values as $\omega_{\mathrm{p}}$ is altered by changing the magnetic flux. The measured $Q F$-values are generally of the order of 0.01-0.1, showing a suppression of almost two orders of magnitude compared to typical values in a similar circuit without special filtering. ${ }^{13}$ This measurement shows that a SQUID-detector can be used for noise spectroscopy of the circuit and the noise source. Results of the present measurements are, however, not yet well characterized. Improved future designs and detectors with higher quality factors should allow to apply the 


\section{Detecting Current Noise with a Josephson Junction}

adiabatic model.

\section{DISCUSSION AND CONCLUSIONS}

The rather strict limits imposed on the adiabatic model can be relaxed by using a more general formalism. To treat the nonequilibrium fluctuations in the setup of Fig. 1 without necessarily requiring adiabaticity, one could approach the problem as quantum tunneling in real time. $\frac{34}{\text { This corresponds }}$ to describing the electrical environment and the fluctuations using an ensemble averaged influence functional and determining the time-evolution of the reduced density matrix of the detector junction. Effects arising from the third and higher moments of fluctuations can then be analyzed by expanding the influence functional in terms of the cumulants of current. On the other hand, the adiabatic or resonant excitation models considered in this work do not take into account the back-action of the detector on the noise source: Instead, the nonequilibrium current fluctuations are considered as if they were caused by an external, independent driving force. This problem can be approached by describing the shot noise source and the detector as a coupled quantum mechanical system in terms of an effective action, which can further be related to the rate of macroscopic quantum tunneling! 11114

To summarize, we have analyzed underdamped Josephson junctions as detectors of current noise. High-frequency fluctuations generated by a shot noise source are described by a thermal activation model with an elevated effective temperature. If the junction stays in its ground state in the absence of transitions induced by high-frequency noise, the rate of macroscopic quantum tunneling is sensitive to the higher moments of fluctuations, too. We have discussed the average escape rate for adiabatic fluctuations which can be directly compared with measurements. The considered experimental scheme allows the detection of non-zero higher odd moments using standard switching measurements provided the high frequencies are efficiently filtered. Relating the measured tunneling rate asymmetries back to the properties of the noise source is a particularly important task, as this involves an accurate characterization of the frequency dependence of the electrical circuit attached to the detector junction.

\section{ACKNOWLEDGMENTS}

We thank J. Ankerhold, O. Buisson, D. Esteve, H. Grabert, T. Heikkilä, F. Hekking, T. Ojanen, H. Pothier, F. Taddei, and J. Vartiainen for useful discussions and O-P. Saira for help in the design of the band-stop filter and 


\section{J.T. Peltonen et al.}

calculation of its frequency response.

\section{REFERENCES}

1. L. S. Levitov, H. W. Lee, and G. B. Lesovik, J. Math. Phys. 37, 4845 (1996).

2. Quantum Noise in Mesoscopic Physics, ed. Yu. V. Nazarov, NATO Science Series in Mathematics (Kluwer, Dordrecht, 2003).

3. B. Reulet, J. Senzier, and D.E. Prober, Phys. Rev. Lett. 91, 196601 (2003).

4. C. W. J. Beenakker, M. Kindermann, and Yu. V. Nazarov, Phys. Rev. Lett. 90, 176802 (2003).

5. Yu. Bomze et al., Phys. Rev. Lett. 95, 176601 (2005).

6. W. Lu et al., Nature 423, 422 (2003).

7. J. Bylander, T. Duty, P. Delsing, Nature 434, 361-364 (2005).

8. S. Gustavsson et al., Phys. Rev. Lett. 96, 076605 (2006).

9. T. T. Heikkilä et al., Phys. Rev. Lett. 93, 247005 (2004).

10. R. K. Lindell et al., Phys. Rev. Lett. 93, 197002 (2004).

11. J. Tobiska, and Yu. V. Nazarov, Phys. Rev. Lett. 93, 106801 (2004).

12. J. P. Pekola, Phys. Rev. Lett. 93, 206601 (2004).

13. J. P. Pekola et al., Phys. Rev. Lett. 95, 197004 (2005).

14. J. Ankerhold, and H. Grabert, Phys. Rev. Lett. 95, 186601 (2005).

15. M. Duckheim, and J. Ankerhold, Phys. Rev. B 71, 134501 (2005).

16. J. Ankerhold, cond-mat/0607020 (2006).

17. Y. M. Blanter, and M. Büttiker, Phys. Rep. 336, 1 (2000).

18. L. S. Levitov, and M. Reznikov, Phys. Rev. B 70, 115305 (2004).

19. J. Salo, F. W. J. Hekking, and J. P. Pekola, Phys. Rev. B 74, 125427 (2006).

20. W. C. Stewart, Appl. Phys. Lett., 12, 277 (1968).

21. D. E. McCumber, J. Appl. Phys. 39, 3113 (1968).

22. K. K. Likharev, Dynamics of Josephson Junctions and Circuits, (Gordon and Breach, New York, 1986).

23. G-L. Ingold, Yu. V. Nazarov, in "Single Charge Tunneling", ed. H. Grabert and M. H. Devoret, NATO ASI Series B, Vol. 294, pp. 21-107 (Plenum Press, New York, 1992).

24. U. Weiss, Quantum Dissipative Systems, (World Scientific, Singapore, 1999).

25. J. Claudon, A. Fay, L. P. Lévy, and O. Buisson, Phys. Rev. B 73, 180502 (2006).

26. J. M. Martinis, and H. Grabert, Phys. Rev. B 38, 2371 (1988).

27. R. J. Schoelkopf et al. in Ref. 2.

28. V. Brosco et al., Phys. Rev. B 74, 024524 (2006).

29. T. Ojanen, and T. T. Heikkilä, Phys. Rev. B 73, 020501 (2006).

30. Higher cumulants can in principle be taken into account in a similar manner, resulting in an asymptotic expansion around a Gaussian distribution. Furthermore, the restriction on $\gamma$ can be avoided by direct numerical inversion of the characteristic function.

31. A. J. Leggett, Phys. Rev. B 30, 1208 (1984).

32. D. Esteve, M. H. Devoret, and J. M. Martinis, Phys. Rev. B 34, 158 (1986).

33. K. E. Nagaev, Phys. Rev. B. 66, 075334 (2002). 


\section{Detecting Current Noise with a Josephson Junction}

34. G. Schön, and A. D. Zaikin, Phys. Rep. 198, 237 (1990). 